\title{
Exploiting SIP for Botnet Communication
}

\author{
Andreas Berger \\ ftw. Telecommunications Research Center Vienna \\ Vienna, Austria
}

\author{
Mohamed Hefeeda \\ School of Computing Science \\ Simon Fraser University \\ Surrey, BC, Canada
}

\begin{abstract}
The Session Initiation Protocol (SIP) implements methods for generic service discovery and versatile messaging. It is, therefore, expected to be a key component in many telecommunication and Internet services. For example, the 3GPP IP Multimedia Subsystem relies heavily on SIP. Given its critical role, ensuring the security of SIP is clearly a crucial task. In this paper, we analyze the SIP protocol and show that it can easily be exploited to mount effective and large-scale botnets. We do this by scrutinizing the details of the SIP protocol and show how it offers a variety of ways to conceal botnet traffic within legitimate-looking SIP traffic. Using our analysis, we implement a SIP bot and present experimental results from a real testbed network. In addition, we employ traffic statistics collected from a large telecommunication provider and discuss the implications for both botnet design and detection. Finally, we present a software tool (called autosip) to generate synthetic traffic that resembles actual SIP traffic with different controllable characteristics. The proposed tool is quite useful for researchers working in the area who may not have access to traffic dumps from actual telecommunication providers.
\end{abstract}

\section{INTRODUCTION}

A bot is a computer running malicious software controlled by a remote adversary. Bots are organized in large groups called botnets. Large aggregation levels of bots can have devastating effects on Internet service availability [1] and are today responsible for the majority of spam [2]. It is hence in the interest of Internet Service Providers (ISPs) to detect botnet activity at an as early state as possible. Botnet communication typically relies on standard Internet protocols like Internet Relay Chat (IRC), HTTP, or P2P file sharing protocols. Communication topologies with a central server component like IRC are simple to implement and offer high stability, but are rather easy to take down by legal authorities. P2P topologies are distributed and hard to disable, but they are more fragile due to peers constantly connecting and leaving the network [3]. Therefore P2P bots have to implement additional communication logic, resulting in more complexity and higher processing requirements. Ideally, a botnet operator would hence aim to have the simplicity and stability of star networks, with the resistivity of P2P networks. As we will show in the following, the infrastructure created using the Session Initiation Protocol (SIP) [4] offers exactly this.

SIP is an important enabler for next generation networks. Most prominently, the 3GPP IP Multimedia Subsystem [5], a replacement for telecommunication operator core networks and a feature-rich service platform, uses SIP as the main signaling protocol. SIP features a generic design and can hence be used for many different purposes like VoIP call control, generic session establishment, asynchronous messaging and even carriage of data. The universal applicability and the large number of existing extensions to the original standard are expected to lead to widespread use of SIP for arbitrary Internet services. However, the diverse nature of SIP traffic can hide malicious activities and the readily available SIP infrastructure offers an ideal alternative for botnet operators. An additional incentive is the universal availability of SIP functionality on many Internet hosts, as modern operating systems are often deployed with preinstalled SIP stacks. Reusing this existing functionality saves the botnet developer time and effort and leads to smaller, less resource intensive bot binaries. Furthermore, as mobile handsets are getting more powerful in terms of processing and networking capabilities, they represent additional attractive targets for future botnets. Many of these devices come with a pre-installed SIP stack too.

In this paper we describe the basic idea of using SIP for botnet communication. We analyze typical botnet requirements and limitations to give guidelines for adequate detection approaches. Section II gives an overview of SIP and shows how bots can exploit the existing infrastructure. We describe the design of our prototypical SIP bot and discuss the generated traffic flow. Learning from these experiences, we recognize that an efficient design must follow a deep understanding of the existing, legitimate SIP traffic. Section III analyzes the dynamics of SIP traffic and shows how legitimate-looking traffic can be created. The benefit of such an investigation is twofold: on the one hand it describes how advanced SIP bots can mimic normal traffic. On the other hand, a tool producing such legitimate background traffic is an important requirement for testing botnet detection approaches in the lab. We present autosip, a tool for emulation of SIP clients, and discuss the collected results. In Section IV, we discuss the related work in the field of botnet analysis. Finally, we summarize our work in Section $\mathrm{V}$ and outline possible future directions.

\section{Analyzing And ExPloiting SIP FOR Botnets}

\section{A. Basic Botnet Functionality}

In the following we outline the basic functionality of botnets. Four types of activity are commonly distinguished:

1) Infection: the initial installation of the botnet malware on the target host. Commonly this happens by tricking the user to run email attachments, by exploiting web browser vulnerabilities, or by attacking the target machine directly using non-patched security holes in the operating system. 
2) Bootstrapping and Maintenance: a bot has to perform a set of procedures to initially find other bots and connect to them. Similarly, it has to be able to counteract when bots disconnect from the botnet. These network maintenance operations are crucial, as only highly available botnets can benefit from their collaborative nature.

3) Command and Control (C\&C): botnet operators need to distribute commands (e.g., start_ddos target $=192.168 .0 .10$ or send_spam rate $=100 /$ hour ) as well as data (e.g., new spam mail templates, bot software updates) to their bots. The bots report current status information back (e.g., how many spam mails were sent in the last 24 hours).

4) Command Execution: in this last phase the bot runs the received commands. This includes starting DDoS attacks and sending spam.

Most existing work in the field of botnets investigates items 1) and 4). Consequently, countermeasures like antivirus software and flooding detectors have been developed. Unlike these approaches, we concentrate on phases 2) and 3). These two phases are responsible for the coordination of attacks and hence represent the fundamental threat that botnets pose. Just like any other network, a botnet has certain requirements in terms of reliability and availability. Given that botnets operate in a hostile environment, stealthyness is a fundamental constraint to be able to achieve these goals. An efficient botnet must continuously find and integrate new bots to grow and compensate for losses. This has to be achieved in a scalable fashion without causing noticeable load neither on the individual infected machines nor on the network. Bots need to be able to locate each other, connect reliably, and communicate with as less required data rate as possible. In the following, we give a short overview of typical SIP functionality and show how SIP matches these requirements.

\section{B. Exploiting SIP for Botnets}

In Fig. 1, a basic (and slightly simplified) SIP session establishment procedure is shown. We assume that caller and callee are already registered to the SIP network. The caller sends a SIP INVITE message to her SIP proxy which in turn looks up the callee's proxy. After being relayed over the two proxies, the message gets delivered to the callee. Consequently, a direct data communication between caller and callee gets established. The currently most common example for this entire operation are VoIP calls, where the caller and callee end up exchanging RTP data with each other. Details regarding SIP procedures can be found in [4].

From the perspective of a botnet operator, there are a number of remarkable features in this basic procedure:

- Once a SIP message is received by the caller's SIP proxy, the SIP infrastructure is responsible for routing it to the recipient. The state of each transaction is monitored by the network and clients are notified whenever the status changes or an error occurs. This allows for more lightweight and hence more stealthy bots.

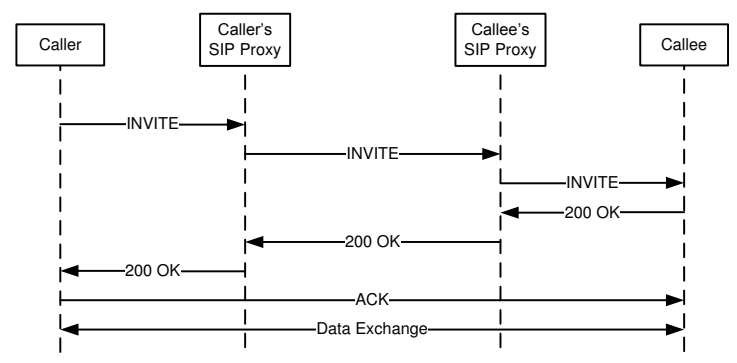

Fig. 1. Basic SIP session establishment.

- Each of the participating hosts must have a port opened to the public Internet, else incoming session establishment requests would fail. These ports cannot be blocked by the network provider without violating the basic SIP requirement of accepting any listening port the client advertises. Given a functional SIP infrastructure, NAT traversal is handled transparently and the bot does not have to deal with it.

- Unlike the commonly exploited IRC service, in SIP there is no rather easily detectable broadcasting of information to all connected hosts. On the other hand and unlike to $\mathrm{P} 2 \mathrm{P}$ protocols, the cost to establish and maintain a dedicated botnet topology is heavily reduced, as the SIP infrastructure was designed to be a stable and reliable signaling platform for arbitrary services.

- SIP proxies are usually message relays and analyze only as much of the semantics of a relayed message as they need for processing it. All other data is considered to be application specific and is usually relayed unmodified. The SIP proxies are not required to be compromised in any way to allow for botnet communication.

- There is both direct and indirect communication foreseen and SIP bots can select their communication strategy from a large variety of options. SIP messages support both user-defined headers as well as virtually arbitrary message bodies. Even in the unlikely event of strict syntax enforcement, bot communication is still feasible as the direct data communication channel can be used.

- Unlike IP addresses, SIP identities do not change when the network connection changes. Infected machines can hence be reliably found and reconnected to the botnet.

In a nutshell, SIP offers a large variety of ways to hide information in legitimate looking traffic. The infrastructure cannot lock out clients easily and no efficient filtering is foreseen. At the same time, it takes full responsibility for delivering messages that are handed to it and informs the client promptly about any problems. In a sense, botnet communication can be considered just another service that uses the existing infrastructure.

\section{Implementation of a SIP Bot}

In order to resemble realistic botnet behavior as closely as possible, we implemented a bot based on the functionality of the well-analyzed Storm bot [6]. Storm employs a modern 
P2P topology based on the Overnet protocol and uses the Kademlia approach [7] for Distributed Hash Tables (DHTs). New commands are published in the P2P network in the same way as data in the equivalent file sharing networks is. Storm bots know how to compute time dependent keys and periodically search for them. As soon as a new command is found, the bot establishes a TCP connection to the particular bot and downloads it.

Overnet uses four main request types: (I) PUBLICIZE to announce the node's presence to the P2P network, (II) CONNECT to establish a connection to a known Overnet peer and to receive a list of other peers, (III) PUBLISH to store a new data item in the network, and (IV) SEARCH to find peers having a specific data item stored. PUBLICIZE requests have an additional role: they are periodically used to ping other known hosts in order to check their availability.

Our implementation adheres to the original functionality but tunnels the entire botnet communication over SIP. The KadC library [8] implements Overnet and supports outgoing requests of all four message types. As there also exists some evidence that this library was used for the development of the original Storm bot [6], we extended it to support incoming requests and modified it to tunnel all Overnet communication over the SIP protocol using the eXosip2 library [9]. Originally, KadC maintains a list of other peers using host identifiers mapped to IP/port combinations. We changed this feature to use a list of SIP URIs in the format sip : $\langle$ user $\rangle @\langle$ domain $\rangle$ instead. Furthermore we removed plenty of NAT support as this is handled transparently by the SIP infrastructure. Note also that in our implementation the bot does not actually download a new command after finding it, but instead just creates a corresponding log message.

Given the numerous ways to establish botnet communication using SIP, we decided not to base our experiments on a specifically optimized SIP communication strategy. Although it is tempting to directly map Overnet peer sessions to SIP sessions, this is only one of a multitude of ways to enable botnet communication. We recognized that it is completely up to the botnet developer what she designates a session in this context. This could either be a traditional SIP session, where peers directly exchange data during the time between the initial INVITE and the final BYE. Alternatively it could be a series of SIP sessions with random length, in order to circumvent detection by analyzing session durations. Furthermore, the SIP MESSAGE extension [10] could be used to reliably deliver messages without establishing a SIP session and therefore with almost no overhead.

Another possibility is to encapsulate the data to be transmitted directly in a standard SIP request, releasing the bots from having to establish a direct data connection. This could be achieved by encoding the data in any of the mandatory header fields that the client has to set (e.g., Call-Id or CSeq), but could also be carried in special user-defined headers. On the other hand, the body of a SIP message can be used, e.g., by encoding the data in a SDP message block. Even worse, all these methods can potentially be combined and picked

\author{
/* SIP bot operation */ \\ Read_contact_list() \\ Run_overnet() /* triggers INVITE transmissions */ \\ while running do \\ Re-register with SIP server \\ message $=$ receive_SIP_message \\ if message $==$ INVITE then \\ Extract message body \\ Hand message body to KadC \\ Send DECLINE \\ end if \\ end while \\ Unregister with SIP server
}

Fig. 2. Pseudocode for the basic functionality of the SIP bot.

randomly whenever a message is to be transmitted.

It is hence pointless to consider a specific communication strategy for the design of a generic SIP bot detection system. As any such system cannot take the format or the rate of SIP messages into account, we chose a simple way of carrying Overnet communication. We setup our SIP bot to encapsulate outgoing messages directly in the body of SIP INVITEs. The receiver sends back a 603 Decline response in order to immediately terminate the SIP session. Note that there is a significant amount of redundancy in this approach, as the body message would not have to carry the client identifier again which is already part of the SIP header. Furthermore, all other SIP messages sent during session establishment (e.g., 100 RINGING, 200 OK, 603 Decline) do not carry any botnet $\mathrm{C} \& \mathrm{C}$ traffic. Developers of real SIP bots would of course optimize the implementation in that regard to minimize the communication overhead and hence render simple rate-based detection approaches ineffective.

Fig. 2 shows the pseudocode of the SIP bot implementation. The SIP bot reads a list of SIP URIs of known bots and runs Overnet to establish connections to them. Botnet messages are extracted from incoming INVITE messages. Whenever a remote bot receives an INVITE message, a DECLINE response is sent to terminate the SIP dialog.

\section{Experimental Evaluation}

We experimented with our implementation of the SIP bot on the network testbed shown in Fig. 3. We ran 30 instances of the SIP bot and monitored the resulting network traffic. Naturally, the net message rate and size just resembled the reported Storm behavior [6], but there is a significant overhead of SIP messages involved. As an example, we discuss in the following the outgoing SIP traffic of a single bot during the first 350 seconds of operation. The bot was initially provided with two SIP identities and was at the end of the monitored period connected to 21 other bots.

Fig. 4(a) demonstrates the 'noisiness' of the bot, with message rates of up to 87 per second. Permanent communication is required to maintain the current connection state. The vast number of SIP messages transmitted by our prototype makes 


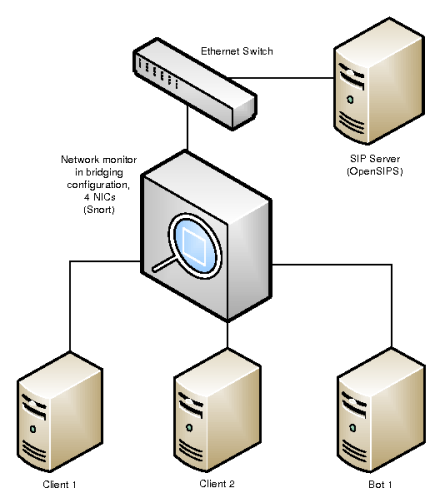

Fig. 3. Testbed setup.

it easily distinguishable from a human user. However, a closer look reveals interesting details: Fig. 4(b) breaks down the types of C\&C messages carried during the time interval shown in Fig. 4(a). Out of 2293 SIP messages in total, only 480 were SIP INVITEs and carried botnet C\&C traffic. Thereof, the main share was the PUBLICIZE requests, followed by CONNECT messages. ${ }^{1}$ The SEARCH messages represent an automatic (unsuccessful) search for a (not existing) command after five minutes of operation.

Overall, we found that developing a more efficient alternative to PUBLICIZE requests would heavily increase Storm's stealthyness. It's network communication is currently dominated by the implemented message pulling paradigm. Each bot has to actively query other bots to learn about their online status and the number of connected hosts. The SIP infrastructure can intuitively be employed to reduce the network load significantly by switching to a message pushing procedure. Two basic ideas are shortly outlined in the following.

- A bot can subscribe to another bot's connection status by using the widely deployed SIP Presence extensions [11]. The SIP infrastructure will in turn send out notifications when the bot disconnects or reconnects. This makes the continuous sending of PUBLICIZE requests unnecessary.

- During a regular SIP session, a bot will be notified about the termination of the connection. Hence, even if SIP Presence cannot be used, the SIP infrastructure can be exploited for monitoring the online status of another bot.

The implementation of any of those two measures would reduce the occurrence of PUBLICIZE requests significantly. At the same time it reduces the complexity of the bot, as some important functionality was offloaded to the SIP infrastructure.

We could have continued here to implement a more efficient communication model for the developed SIP bot. However, we do not intend to propose the ultimate SIP C\&C protocol but are particularly interested in the traffic caused by such stealthy bots, in order to learn about the requirements of a adequate

${ }^{1}$ Due to low total number of bots available in our experiment, the bot never ended up being sufficiently connected to stop connection attempts. In a realworld network (like Overnet), the phase where large bursts of CONNECT requests are seen ends shortly after the peer starts running [6].

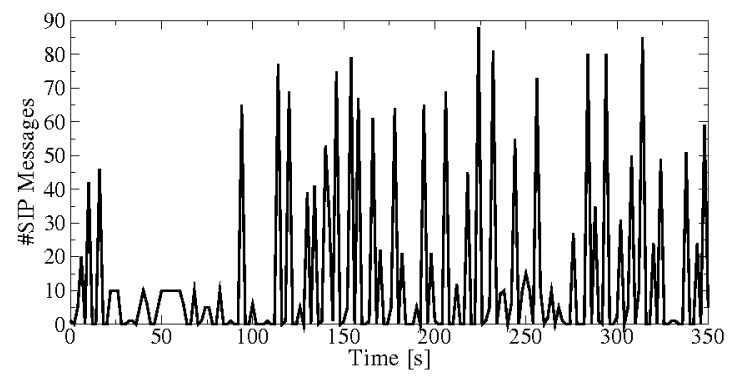

(a) Distribution of outgoing SIP messages produced by one bot instance in the first 350 seconds after activation.

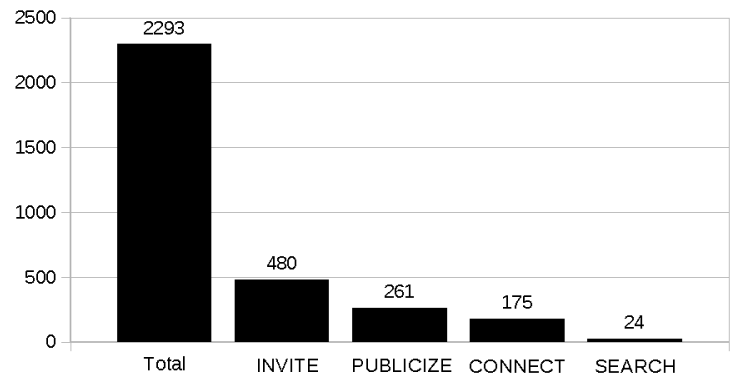

(b) Distributions of the total number of sent messages, number of INVITE messages, and types of included botnet communication.

Fig. 4. Experimental results from running our SIP bot.

detection approach. The detectability of a SIP botnet depends heavily on the properties of legitimate SIP traffic in the network. E.g., an ideal communication strategy in a network with plenty of MESSAGE requests originating from legitimate users would adapt this behavior and base the communication on this type of request. It is not obvious how much SIP traffic there is in a network and how it is composed. Even more importantly, the dynamics of the background traffic have to be taken into account. Stealthy bots are expected to react on otherwise quiet phases and reduce their communication, e.g., at night.

\section{UNDERSTANDING SIP TRAFFIC}

\section{A. Analyzing Real SIP Traffic}

A fundamental requirement for evaluating new SIP bot detection approaches is a solid understanding of regular and legitimate SIP activities in a network. IP traffic is generally highly dynamic and strongly varies with the time of day. Detection algorithms must be able to take this varying baseline scenario into account in order to achieve reasonable detection rates while producing few false alarms. As an example, Fig. 5 (a) shows the varying distribution of users connected to a mobile network. ${ }^{2}$ While there is almost no activity to be expected at five $\mathrm{AM}$, the network load peaks at around nine PM. Each day is different from each other, and weekends are significantly different from weekdays. Both SIP bots as well as detection approaches need to take this into account.

\footnotetext{
${ }^{2}$ These data originate from a large European mobile operator and were collected during a previous project.
} 


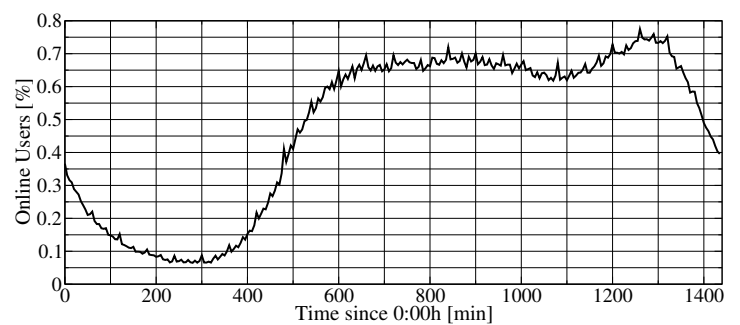

(a) Distribution of online users in a live $3 \mathrm{G}$ network during a weekday. It covers 24 hours and starts at 0:00h.

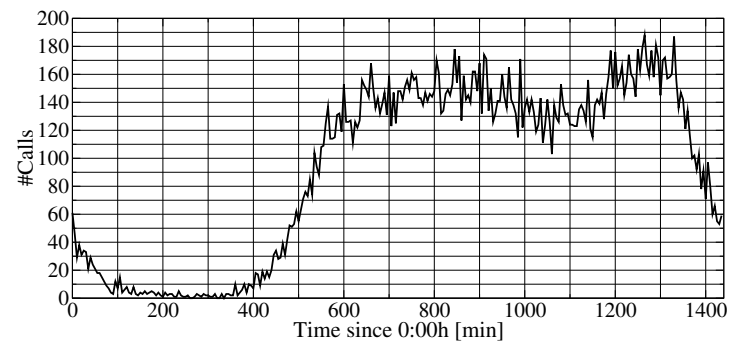

(b) Traffic emulation results of autosip. Parameters: $N=1000$; $X=20 ; \alpha=0.75 ; c=6 ; \mu=4 ; \sigma=1$; and bin size $=5 \mathrm{sec}$.

Fig. 5. Traffic generated by autosip vs. real traffic.

\begin{tabular}{|l|c|c|}
\hline Average number of call attempts per hour and user & $c$ & 6 \\
Mean value of call duration & $\mu$ & 4 \\
Standard deviation of call duration & $\sigma$ & 1 \\
Number of simulated SIP clients & $N$ & 1000 \\
Number of friends of each client & $X$ & 15 \\
Probability of calling a friend & $\alpha$ & 0.8 \\
\hline
\end{tabular}

TABLE I

AUTOSIP TRAFFIC EMULATION PARAMETERS AND TYPICAL VALUES.

We have identified a set of traffic features to be relevant for the emulation of the defined SIP scenario. The given values exemplify realistic SIP traffic and its dynamics, but are not meant to represent all possible traffic combinations or applications. Due to a lack of representative SIP traffic traces, we base our experiments on session features as they are known from phone calls. This is driven by the fact that VoIP currently represents SIP's most prominent application and matches the communication strategy (using INVITEs) we adopted in Section II-C. We emphasize that SIP bot detection approaches must be able to work in presence of any other future application scenario, for which we today cannot determine and emulate the characteristic session features. The setup described in the following aims to provide a realistic and, most notably, highly dynamic environment instead of wrongly assuming static traffic features. The following scenario parameters are taken into account and are summarized in Table I:

- Number of online users: As we have already discussed, the number of online users varies with time. Our emulation setup follows real-world user distributions and automatically varies the number of connected users with time.

- Call duration: The duration of voice calls can be modeled with a lognormal distribution. In [12] the authors verify

\author{
/* autosip client main operation */ \\ Connect to manager \\ while not quit do \\ Wait for execute command \\ Receive scenario parameters \\ Read contacts file and pick $X$ friends \\ while running do \\ Re-register with SIP server \\ Answer incoming calls \\ if not call running AND $i s_{-}$time_for_call $(c)$ then \\ Send INVITE to get_contact $(\alpha)$ \\ Hold call for get_lognormal_sample $\left(\mu_{L}, \sigma\right)$ \\ end if \\ end while \\ Unregister with SIP server

\section{end while}

Fig. 6. Pseudocode for the basic functionality of the autosip client.

this assumption and provide figures for the distribution parameters $\mu$ and $\sigma$.

- User contacts: Phone customers typically do not call every other user with the same probability. To model that behavior, we assume that any given user would call $X$ friends out of $N$ contacts with probability $\alpha$ and would contact any of the remaining $N-X$ users with probability $1-\alpha$.

- Call rate: The total number of calls in a telephone network is related to the total number of connected users at a particular instance in time. We assume that each connected user makes on average $c$ call attempts per hour. Based on this, a uniform call probability per minute $\frac{c}{60}$ is derived. Note that in our scenario call attempts fail when callees are either not online or busy.

\section{B. Generating Synthetic SIP Traffic}

Our tool, autosip, for emulation of realistic SIP traffic consists of a client and a server component. The clients implement the eXosip SIP stack that enables them to send and accept SIP INVITE requests. Furthermore, additionally required signaling like SIP registration, acknowledgments, and status message handling is carried out. As already noted in the introduction, all of the described features can also be used for the design of stealthier bots.

Fig. 6 describes the basic functionality of the autosip client. Upon start, each client picks $X$ random friends from this list and registers with the SIP server. It begins then to try to initiate calls to other clients. On average, $c$ call attempts per hour are made. If a call attempt is accepted by the callee, the caller computes a call duration using parameters $\mu$ and $\sigma$. As soon as the computed duration is over, the caller terminates the call. There can always be only a single ongoing call per client. During an ongoing call, the client does not make call attempts and answers incoming call attempts with a SIP BUSY message. Each client periodically sends re-registration requests to the SIP server in order to avoid the session to time out. 
The server component of autosip is called the manager. It controls the operation of the clients, sets and distributes the scenario parameters (see Table I), and collects the individual results. It automatically varies the number of active clients with time, following a given client distribution.

Fig. 5 shows an example result for the number of calls made over time using the also shown distribution of online users as input. These results can be interpreted in two ways: autosip can be used in the lab to evaluate anomaly detection algorithms for SIP. The second learning is that bots can simply resemble what autosip does and make their communication stealthier. Given that the development of such advanced bots is clearly feasible (as most of the hard work is carried out by the SIP infrastructure), it is challenging to come up with adequate countermeasures. In the following, we give a short overview of the existing work in this field.

\section{RELATED WORK}

The analysis of botnet communication is a rather new field and limited prior work is available. Strayer et al. give in [13] a comprehensive overview of the current state of the art. While their own work and experiments are targeted exclusively at detection of IRC based botnets, the discussion of their classifier and correlation stages are universally relevant. Botminer is a recent proposal by $\mathrm{Gu}$ et al. [14]. Their main contribution is the correlation of results from the analysis of the botnet activity (A-plane) and the C\&C traffic (C-plane). Based on that, their proposal can detect IRC, HTTP, and P2P botnets. The core input data in use are called $C$-flows, consisting of several average statistics for the number of flows per hour, the number of packets, etc. The proposed system does not take sequences of events into account, but analyzes each event independently. Gu et al. propose BotSniffer [15], a system for detection of botnet C\&C traffic over IRC and HTTP. The approach is based on detecting dense crowds of hosts which send homogeneous messages and is hence applicable only to botnets with a centralized communication topology. An interesting result by Dagon et al. [16] shows how the number of online bots depends on their location and the local time. These findings emphasize the importance of taking dynamic traffic features into account.

Other approaches try to disrupt botnet communication by transmitting bogus $\mathrm{C} \& \mathrm{C}$ messages. E.g., Holz et al. demonstrate in [17] an effective way to pollute the Storm botnet using custom bot software to render it inefficient. Similar approaches can certainly be mounted against SIP-based botnets, but are no substitute for effective network monitoring. Due to the large variety of $\mathrm{C} \& \mathrm{C}$ protocols that can be established on top of SIP, only thorough traffic inspection can detect the abuse of SIP infrastructures.

\section{COnClusions And Future Work}

We analyzed the SIP protocol and its potential exploitation by botnet operators. We presented a simple method for the creation of SIP-based botnets and outlined an actual implementation of a SIP bot. Overall, the development of a SIP based bot turned out to be even simpler than we expected. The presented SIP-based botnet, although simple, will hardly be detected by any SIP intrusion detection system and might in the best case be wrongly recognized as a flooding attack. Since SIP is expected to be a fundamental Internet service enabler, the increased traffic will help to conceal botnet communication. We conclude that SIP is a highly attractive platform for botnets, because it is readily available and by definition open for everyone. Next steps of this research include the development of a bot with a communication strategy that fits the SIP architecture better than Storm and that exploits available SIP services like Presence and MESSAGE. Such an advanced bot can consequently be used to evaluate new monitoring and detection approaches for SIP platforms.

\section{REFERENCES}

[1] S. Berinato, "How a bookmaker and a whiz kid took on a DDOS-based online extortion attack," May 2005. [Online]. Available: http://www.csoonline.com/article/220336/How_a_Bookmaker_ and_a_Whiz_Kid_Took_On_a_DDOS_based_Online_Extortion_Attack

[2] Y. Zhao, Y. Xie, F. Yu, Q. Ke, Y. Yu, Y. Chen, and E. Gillum, "BotGraph: large scale spamming botnet detection," in Proc. of the USENIX Symposium on Networked Systems Design and Implementation, Boston, MA, 2009.

[3] D. Stutzbach and R. Rejaie, "Understanding churn in peer-to-peer networks," in Proc. of the ACM SIGCOMM Conference on Internet Measurement, Rio de Janeriro, Brazil, 2006, pp. 189-202.

[4] J. Rosenberg, H. Schulzrinne, G. Camarillo, A. Johnston, J. Peterson, R. Sparks, M. Handley, and E. Schooler, SIP: Session Initiation Protocol. IETF, RFC 3261.

[5] G. Camarillo and M. Garcia-Martin, The 3G IP Multimedia Subsystem (IMS): Merging the Internet and the Cellular Worlds. John Wiley \& Sons, Aug. 2004.

[6] F. Dahl, "Der Storm-Worm," Master's thesis, University of Mannheim, Mar. 2008.

[7] P. Maymounkov and D. Mazires, "Kademlia: A Peer-to-Peer information system based on the XOR metric," in Proc. of the International Workshop on Peer-to-Peer Systems. Cambridge, MA: Springer-Verlag, 2002, pp. 53-65.

[8] "KadC - P2P library." [Online]. Available: http://kadc.sourceforge.net

[9] A. Moizard, "The eXtended osip library." [Online]. Available: http://savannah.nongnu.org/projects/exosip

[10] B. Campbell, J. Rosenberg, H. Schulzrinne, C. Huitema, and D. Gurle, Session Initiation Protocol (SIP) Extension for Instant Messaging. IETF, RFC 3428.

[11] J. Rosenberg, A Presence Event Package for the Session Initiation Protocol (SIP). IETF, RFC 3856.

[12] G. Boggia, P. Camarda, A. D’Alconzo, A. D. Biasi, and M. Siviero, "Drop call probability in established cellular networks: from data analysis to modelling," in Proc. of the Vehicular Technology Conference, vol. 5, Stockholm, Sweden, 2005, pp. 2775-2779.

[13] W. Strayer, D. Lapsely, R. Walsh, and C. Livadas, Botnet Detection Based on Network Behavior, ser. Advances in Information Security. Springer-Verlag, 2008, vol. 36, pp. 1-24.

[14] G. Gu, R. Perdisci, J. Zhang, and W. Lee, "BotMiner: clustering analysis of network traffic for protocol- and structure-independent botnet detection," in Proc. of the USENIX Security Symposium, San Jose, CA, 2008, pp. 139-154.

[15] G. Gu, J. Zhang, and W. Lee, "BotSniffer: detecting botnet command and control channels in network traffic," in Proc. of the Network \& Distributed System Security Symposium, San Diego, CA, 2008.

[16] D. Dagon, C. Zou, and W. Lee, "Modeling botnet propagation using time zones," in Proc. of the Network and Distributed System Security Symposium, San Diego, CA, 2006.

[17] T. Holz, M. Steiner, F. Dahl, E. Biersack, and F. Freiling, "Measurements and mitigation of peer-to-peer-based botnets: a case study on storm worm," in Proc. of the 1st USENIX Workshop on Large-Scale Exploits and Emergent Threats, San Francisco, California, 2008, pp. 1-9. 